CoV-2 infection in residents of a large homeless shelter in Boston. JAMA. 2020; 323: 2191-2192.

5. Advisory for managing Health care workers working in COVID and non-COVID areas of the hospital. Accessed on October 22, 2020. Available from https://www.mohfw.gov.in/pdf/Advisory formanagingHealthcareworkersworkinginCOVIDandNon COVIDareasofthehospital.pdf

6. Fifth of Chennai residents exposed to Covid: Survey. Accessed on November 20,2020 Available from https://www.hindustantimes. com/india-news/fifth-of-chennai-residents-exposed-to-covidsurvey/story-bYJePr6f TEsqrNh8VB3c4I.html
7. Infection prevention and control during health care when novel coronavirus (nCoV) infection is suspected. World Health Organization. Accessed on December 23, 2020. Available from: https:// www.who.int/publications-detail/infection-preventionand-control-during-health-care-when-novel-coronavirus-(ncov)infection-is-suspected-20200125

8. Hunter BR, Dbeibo L, Weaver C, et al. Seroprevalence of severe acute respiratory coronavirus virus 2 (SARS-CoV-2) antibodies among healthcare workers with differing levels of coronavirus disease 2019 (COVID-19) patient exposure. Infect Control Hosp Epid. 2020;41:1441-42.

\section{Digestive Tract Injuries Caused by Ingested Foreign Bodies Containing Magnets}

We report on 16 children with ingestion of magnetic foreign bodies, who were identified by a medical record review of our hospital data for the time period between January, 2017 and May, 2018. Digestive tract wall was sandwiched in $13(75 \%)$ children and 11 (74\%) had gaptic intestinal perforation.

Keywords: Toys, Unintentional injuries.

The ingestion of magnetic foreign bodies in children often requires urgent management, which is quite often surgical [1]. A retrospective analysis was conducted on 16 patients (10 males), who were admitted to our hospital from January, 2017 to May, 2018, and presented with digestive tract injuries caused by magnetic foreign bodies ingestion. The age of these patients ranged within 2-9 years old (median: $4.6 \pm 0.5$ years old), with 6 $(37.5 \%)$ infants and 6(37.5\%) infants.

An abdominal $X$-ray was used to detect the foreign bodies. Appropriate measures were taken for the removal of these foreign bodies. Two patients had removal using foreign body forceps, three patients underwent removal surgery with the use of magnetic attraction, (cylindrical magnets were bound at the end of the snare), and 11 patients underwent laparotomy and repair of the digestive tract.

The foreign bodies were all magnetic components, and $50 \%$ of these foreign bodies were buckyballs. The digestive tract walls of 13 patients $(81.2 \%)$ were sandwiched by the attraction of magnetic foreign bodies, and were injured. Gastric intestinal perforations presented in 11 patients $(68.8 \%)$, while one patient had 20 magnetic foreign bodies located in seven different sites of the small intestine, causing seven perforations in the small intestine.

Abdominal pain $(31.2 \%)$, vomiting $(12.5 \%)$, or both $(37.5 \%)$ were the commonest complaint. In one patient, a tiny "gap" (Fig. 1) in the middle of the foreign body was noted by $X$ ray, and the small intestine walls were sandwiched by the attraction of two small magnetic foreign bodies, resulting in the perforation of the small intestine. All patients had a satisfactory outcome. Five patients underwent gastroscopic removal of foreign bodies and 11 patients underwent laparotomy for removal surgery.
With magnetic foreign bodies, gastrointestinal pressure necrosis between foreign bodies and the formation of fistula can occur [2]. The diagnosis of magnetic foreign bodies in the digestive tract is mainly dependent on the medical history, and the abdominal $X$-ray. Both anteroposterior and lateral $X$-ray films of the abdomen must be combined with careful examination, in order to determine whether a tiny 'gap' in its middle is present for the single metal foreign body in a fixed position of the digestive tract. Therefore, for multiple small adhesive metal foreign bodies revealed by an abdominal $X$-ray film, it should first be considered whether these are connected by the attraction among these magnetic foreign bodies, and whether the digestive tract walls are sandwiched by the attraction of these foreign bodies.

Upon magnetic approximation (when more than one foreign body is ingested), a considerable amount of force can result in inseparable magnetic attraction between bowel loops [3], which would rapidly result in the necrosis and perforation of the intestines.

Conservative treatment may be appropriate for patients who have ingested a single foreign body [4,5]. Although foreign body forceps under a gastroscope cannot effectively grasp these foreign bodies, based on the experience of the investigators, this can be performed as long as the connection point between the two magnetic foreign bodies formed by the magnetic attraction is grasped by the foreign body forceps.

Magnetic foreign body ingestion has typical features on the abdominal $X$-ray and early laproscopic/surgical intervention leads to a good outcome.

Ethics approval: Ethics Committee of The General Hospital of Ningxia Medical University; No. 2019-388.

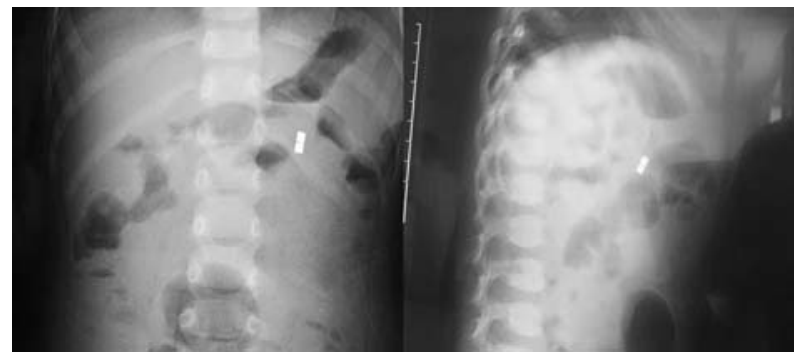

Fig. 1 The anteroposterior and lateral $X$-ray films of the abdomen, showing a 'gap' among the small magnetic foreign bodies. 
Contributors: ZLS: drafting the manuscript and revising it critically for important intellectual content; ZCS,SZH: substantial contributions to conception and design of the work; DL,XHZ,XET: substantial contributions to the acquisition, analysis, and interpretation of data for the work. All authors approved the final version of manuscript, and are accountable for all aspects related to the study.

Funding: None; Competing interest: None stated.

Published online: June 24, 2020; PII: S097475591600205

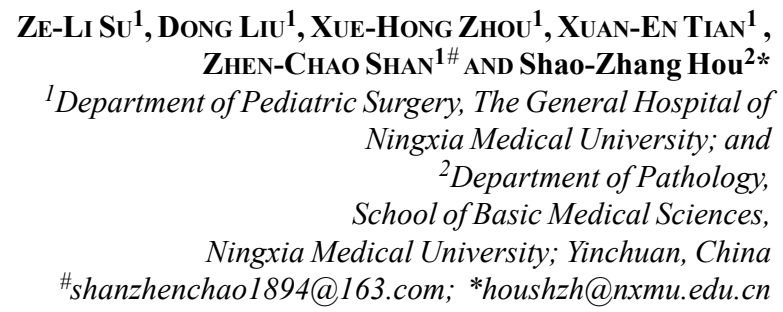

\section{REFERENCES}

1. Talvard M, Mouttalib S, Flaum V, Viala J, Galinier P, Olives JP, et al. [Magnet ingestions in children: A French multicenter study]. Arch Pediatr. 2015;22:32-8. [Article in French]

2. Lee WH, Min YD, Moon KR. A case of gastroduodenal fistula caused by ingested magnetic foreign bodies. Korean J Pediatr Gastroenterol Nutr. 2008;11:84-8.

3. Brown JC, Otjen JP, Drugas GT. Pediatric magnet ingestions: The dark side of the force. Am J Surg. 2014;207:754-9.

4. Tavarez MM, Saladino RA, Gaines BA, Manole MD. Prevalence, clinical features and management of pediatric magnetic foreign body ingestions. J Emerg Med. 2013;44: 261-8.

5. Butterworth J, Feltis B. Toy magnet ingestion in children: Revising the algorithm. J Pediatr Surg. 2007;42:e3-e5.

6. Hussain SZ, Bousvaros A, Gilger M, et al. Management of ingested magnets in children. J Pediatr Gastroenterol Nutr 2012;55:239-42.

\section{Pediatric ABO-incompatible Living Related Donor Liver Transplantation: Experience from Indian Subcontinent}

We present our experience with pediatric $\mathrm{ABO}$-incompatible liver transplantation in India. Data of patients $<18$ years of age undergoing $\mathrm{ABO}$-incompatible liver transplantation our hospital between January, 2011 and November, 2018 were analyzed. Plasmapheresis was done pre-transplant till antibody titer was $<16$ units. Rituximab/Intravenous immunoglobulin was used for immunosuppression, in addition to standard drugs (mycophenolate mofetil, steroids, and tacrolimus). Out of 203 patients that underwent liver transplant during this period, 8 underwent $A B O$-incompatible liver transplantation; 4 ( 3 boys) had blood group O+ve. Median (range) age was 28 (7-91) mo, PELD score was 24.5 (14-42), and pre-transplant antibody titer range was 1:32-1024. Number of plasmapheresis sessions required ranged from 1-6. Post-operatively two patients had rise in antibody titer $>64$ requiring plasmapheresis. All 8 patients survived without rejection/biliary issues. Mean (range) of posttransplant hospital stay was 19.1 (13-22) $\mathrm{d}$ and follow-up period was 38.1 (7.1-84.4) mo. Pediatric ABO-incompatible liver transplantation can be successfully performed using plasmapheresis with optimal immune-suppression and vigilant post-op monitoring.

Keywords: Immunoadsorption, Outcome, Plasmapheresis, Rituximab.

Due to shortage of cadaveric organ donation, living donor liver transplantation (LDLT) is the primary form of liver transplantation (LT) in India. In the LDLT scenario, donors are restricted to family members and it is not always possible to find a healthy blood group-compatible donor in time. Liver transplant across the ABO blood group barrier is a promising approach in such patients. Antibody-mediated rejection along with biliary and vascular complications are the usual limiting factors in ABO-incompatible (ABOi) LT [1]. Determining optimal immunosuppression to avoid complications in ABOi LT is challenging. Desensitization with use of plasmapheresis and anti-CD20 monoclonal antibody (rituximab) plays an important role in successful outcome of ABOi LT [2]. Our aim was to study the course and outcomes of pediatric ABOi LT.

Data of all patients $(<18$ years) undergoing ABOi LDLT at our hospital, India between January, 2011 and November, 2018 were retrospectively analyzed. ABOi LT was performed only in cases where compatible related donors were unavailable even after exploring option of swap transplant between two families. Informed consent was taken for ABOi LDLT. Hospital liver transplant committee clearance was obtained. Demographic details, primary diagnosis and severity of liver disease using Pediatric end-stage liver disease (PELD) score were noted. Recipient and donor blood group and pre-transplant antibody titres were recorded. Surgical details and post-operative course including antibody titres, duration of ICU and hospital stay, incidence of hemolysis, rejection, biliary and vascular issues and infections were recorded.

For immunosuppression, all patients were started on mycophenolate mofetil (MMF) one week pre-transplant. After 2016, rituximab was added to the institutional protocol for pretransplant preparation CD19 levels were in those receiving rituximab monitored before and post LT. Plasmapheresis was done pre-LT on alternate days to reduce antibody titre16. Prior to 2012, conventional plasmapheresis with AB blood group fresh frozen plasma was done. Thereafter with the availability of 2A column filter (Evaflux 2A column; Kawasumi Laboratories) at our institution, cascade plasmapheresis was performed. In cases where conventional/cascade plasmapheresis failed to decrease antibody titre $<16$ or when urgent LT was needed, plasmapheresis with immune-adsorption technique using Glycosorb/ Adsopak filter was done. Prior to 2016, intravenous immunoglobulin (IVIG) was given for first five days post-operation to prevent antibody-mediated rejection. After 2016, IVIG was no longer used prophylactically, but reserved only for treatment of antibody-mediated rejection. Post-operatively triple immunosuppression with MMF, steroids and tacrolimus was administered. Target trough level of tacrolimus was $10-12 \mathrm{ng} / \mathrm{mL}$ in the first month post-LT. Antibody titers were closely monitored and the threshold to do plasmapheresis was a titer $\geq 64$ for up to two weeks post-LT. 